\title{
Dynamical Modeling of COVID-19 and Use of Optimal Control to Reduce the Infected Population and Minimize the Cost of Vaccination and Treatment
}

\author{
Yohannes Dhimas Mahardika* \\ Department of Mathematics, Diponegoro University (UNDIP) \\ Jln. Prof. Soedarto, SH., Semarang 50275, Indonesia \\ dhimasmahardika@student.undip.ac.id
}

Received: $22^{\text {nd }}$ May 2020/ Revised: $2^{\text {nd }}$ March 2020/ Accepted: $2^{\text {nd }}$ March 2021

\begin{abstract}
How to Cite: Mahardika, Y. D. (2021). Dynamical Modeling of COVID-19 and Use of Optimal Control to Reduce the Infected Population and Minimize the Cost of Vaccination and Treatment. ComTech: Computer, Mathematics and Engineering Applications, 12(2), 65-73. https://doi.org/10.21512/comtech.v12i2.6466
\end{abstract}

\begin{abstract}
The research described a model formulation of COVID-19 using a dynamic system of Ordinary Differential Equation (ODE) which involved four population systems (susceptible, exposed, infectious, and recovered). Then, the research analyzed the direction of the equilibrium, Disease Free Equilibrium (DFE), and Endemic Equilibrium (EE). The treatment and vaccination were the control functions applied to the dynamical system modeling of COVID-19. The research was done by determining dimensionless number $\mathrm{R}_{0}$ or Basic Reproduction Number and applying optimal control into the dynamical system using the Pontryagin Minimum Principle. Numerical calculations were also performed to illustrate and compare the graph of the dynamical system with and without a control function. From the results, there is a reduction in the number of susceptible and infected populations. It indicates that giving vaccines to susceptible populations and treating infected populations affect the number of susceptible and infected populations. It also means that this control can reduce the spread of the virus.
\end{abstract}

Keywords: dynamical modeling, optimal control, infected population, cost of vaccination, treatment, COVID-19

\section{INTRODUCTION}

The instability of economic growth becomes a serious discussion for many companies in the world, especially manufacturing or service company. Because of that situation, the company needs to anticipate it by maintaining its operation. Many ways can be done by the company to survive and maintain its continuity of business operations. One of the ways is closely monitoring productivity and quality of products.

In recent years, the use of dynamical systems in biology has shown significant changes in mathematics and biosciences (Brauer \& Castillo-Chavez, 2013). This dynamical system uses the optimal control laws to look for optimization of functional objectives for a certain period and find the optimum control of the dynamical system. The use of this optimum control is expected to be seen in the graphic. Later, it will show the difference between a dynamical system that uses and does not use control.

At the end of 2019, WHO was informed by the Chinese government about several cases of pneumonia with unfamiliar etiology. As an emerging business hub of China, Wuhan experienced an outbreak of a novel coronavirus that killed more than eighteen hundred and infected over seventy thousand people within the first fifty days of the epidemic. The outbreak was initiated from the Hunan seafood market in Wuhan, China and rapidly infected more than 50 people. The frequently sold animals at the Hunan seafood market were bats, frogs, snakes, birds, marmots, and rabbits (Wang, Horby, Hayden, \& Gao, 2020).

This virus is reported to be a member of the b-group of coronaviruses. The novel virus is named Wuhan coronavirus or 2019 novel coronavirus (2019nCov) by Chinese researchers. The International Committee on Taxonomy of Viruses (ICTV) mentions the virus as SARS-CoV-2 and the disease as COVID-19 (Shereen, Khan, Kazmi, Bashir, \& Siddique, 2020).

The source of origin and transmission is important to determine and develop preventive strategies to contain the infection. In the case of SARS-CoV, the previous research initially focuses on raccoon dogs and palm civets as key reservoirs of 
infection. However, only the isolated samples from the civets at the food market show positive results for viral RNA detection. It suggests that the civet palm might be the secondary host (Li, Chen, \& Huang, 2020). In another previous research, the samples are healthy people in Hongkong, and the molecular assessment shows $2,5 \%$ of the frequency rate of antibodies against SARS-coronavirus. These indications suggest that SARS-coronavirus may have been circulating in humans before causing the outbreak in 2003 (Zheng et al., 2004). Later on, Rhinolophus bats are also found to have anti-SARS-CoV antibodies suggesting the bats as a source of viral replication (Wang \& $\mathrm{Hu}, 2013$ ).

It is necessary to know that to avoid coronavirus infection, people need to maintain a distance between sick people at least $2 \mathrm{~m}$ or more. Until now, there is no research that states coronavirus can be transmitted by air. The virus can spread easily through small droplets from the nose or mouth of infected people when they sneeze or cough. Moreover, the droplets from infected people can land on objects or surfaces and are touched by healthy persons who further touch their eyes, nose, or mouth (Annas, Pratama, Rifandi, Sanusi, \& Side, 2020).

The research applies a fixed time and free endpoint for optimal control. The purpose is to predict the dynamic population of susceptible, exposed, infected, and recovered people for a certain time using the dynamical system of Ordinary Differential Equation (ODE) modeling and the effect of vaccination and treatment as a control in the model. The reason to conduct the research is that the dynamical modeling system of ODE is suitable to describe the characteristic of the spread of contagious disease in a certain population.

\section{METHODS}

The compartmental model simplifies a mathematical modeling of infectious diseases. The population is assigned to compartments with labels or classes. For example, the research applies Susceptible, Infectious, or Recovered (S, I, or R). People may progress between compartments. The order of the labels or class usually shows the flow patterns between the compartment, for example, SEIS means susceptible, exposed, infectious, and susceptible again (Krishna \& Prakash, 2020).

The dynamical modeling is most often run with ordinary differential equations (which are deterministic). However, it can also be used with a stochastic (random) framework. The framework is more realistic but more complicated to analyze. The model also tries to predict things such as how a disease spreads, how much the total infected number is, or how long the duration of an epidemic is. It also estimates various epidemiological parameters, such as the reproductive number. Then, it can show how different public health interventions may affect the outcome of the epidemic, for example, it indicates the most efficient technique for issuing a limited number of vaccines in a given population (Krishna \& Prakash, 2020).

A classic epidemiological model to study the dynamics of an infectious disease is the Susceptible (S) - Exposed (E) - Infectious (I) - Recovered (R) model. This SEIR model establishes the most recent epidemiological data of the COVID-19 outbreak in China. The transmission rate, $\beta$, controls the spread rate, representing the probability of transmitting disease between a susceptible and an infectious individual. Meanwhile, the incubation rate, $\gamma$, is the rate of latent individuals becoming symptomatic (the average incubation duration is $1 / \gamma$ ). Then, the probability of recovery, $\theta$, is the average rate of recovery and infected populations (Krishna \& Prakash, 2020).

The classic SEIR equation assumes a constant Susceptible (S) of population size with constant birth and death rate across all compartments. In the actual situation, this population is dynamic. There will be a large number of people moving in and out of each city and epidemic-associated deaths (Yang et al., 2020).

In the research, there are several stages. First, the research divides the total population into four compartments or classes: $\mathrm{S}$ for susceptible population, $\mathrm{E}$ for latent or exposed population, I for infected population, and $\mathrm{R}$ for recovered population. The flowchart diagram for the compartments can be seen in Figure 1.

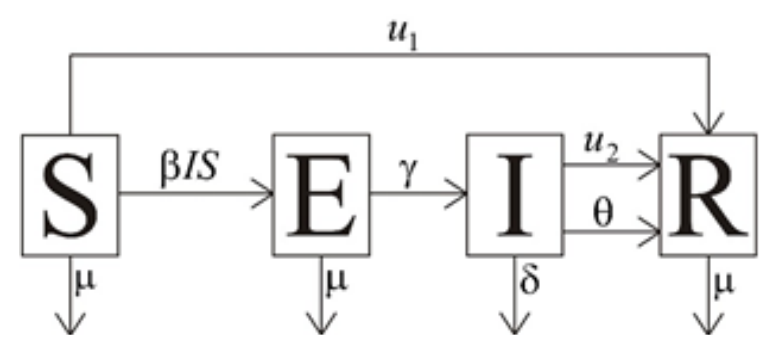

Figure 1 Flowchart Diagram of COVID-19

Second, the Basic Reproduction Number $\left(R_{0}\right)$ is determined, and the system equilibrium is analyzed. Third, the research forms the optimal control using Pontryagin minimum principle with the objective function of minimizing the susceptible, infected population (compartment), and value (cost) of vaccine and treatment. Fourth, numerical analysis of the optimal control is calculated with GNU Octave software (Eaton, Bateman, Hauberg, \& Wehbring, 2021) and using the forward-backward sweep of the fourth-order Runge Kutta method. Forward of fourthorder Runge Kutta method is for solving the state in Equation (6), and backward in fourth-order Runge Kutta method is for adjoint variable in Equation (9) (Anita, Arnăutu, \& Capasso, 2011). 


\section{RESULTS AND DISCUSSIONS}

Dynamical modeling of coronavirus spread is based on Figure 1.

$$
\begin{aligned}
& \frac{d S}{d t}=r N_{\sigma}-\frac{\beta I S}{N_{\sigma}}-\mu S \\
& \frac{d E}{d t}=\frac{\beta I S}{N_{\sigma}}-\gamma E-\mu E \\
& \frac{d I}{d t}=\gamma E-\theta I-\delta I-\mu I \\
& \frac{d R}{d t}=\theta I-\mu R
\end{aligned}
$$

$0, R(0)>0$

With initial condition $S(0)>0, E(0)>0, I(0)>$

This SEIR dynamical system model in Equation (1) is based on Yang et al. (2020). Then, it is modified by giving the terms of natural death rate $(\mu)$, death rate caused by infection $(\delta)$, and source of susceptible rate $\left(r N_{\sigma}\right)$. The $\left(r N_{\sigma}\right)$ is source rate of susceptible population, $\mu$ is parameter of natural death rate, and $\gamma$ is parameter of transmissions rate from exposed individuals in becoming infected individuals. Moreover, $\theta$ is the parameter of recovery rate from infection to recovered, $\delta$ is the parameter of death rate caused by infection of the virus, and

$$
\frac{\beta I S}{N_{\sigma}}
$$

is function of suspected individual moving to the exposed compartment with parameter rate $(\beta)$. The rate of changed population of susceptible, exposed, infected, and recovered represent $S, E, I, R$ respectively.

To analyze the equilibrium point, the research needs to calculate and determine Basic Reproduction Number $\left(R_{0}\right)$, Disease Free Equilibrium (DFE), and Endemic Equilibrium (EE). In here, $R_{0}$ is defined as the expected number of secondary infections produced by a single infection in a completely susceptible population. Basically, $R_{0}$ is used to determine the spread level of the disease. One of the most important concerns about infectious diseases is their ability to invade a population. The Basic Reproduction Number, $R_{0}$, is a measure of the potential for disease spread in a population and is inarguably one of the foremost and most valuable ideas that mathematical thinking has brought to epidemic theory (Hattaf \& Dutta, 2020). It represents the average number of secondary cases generated by an infected individual if it is introduced into a susceptible population with no immunity to the disease in the absence of interventions to control the infection.

If it is $R_{0}<1$, on average, an infected individual produces less than one newly infected individual over the course of his/her infection period. In this case, the infection may die out in the long run. Conversely, if it is $R_{0}<1$, each infected individual produces, on average, more than one new infection. The infection will spread in a population. A large value of $R_{0}$ may indicate the possibility of a major epidemic (Delamater, Street, Leslie, Yang, \& Jacobsen, 2019).

The idea of $R_{0}$ is developed by Van den Driessche (2017) and Kumar and Kumar (2018). Basically, $R_{0}$ is used to determine the spread level of the disease. It can be determined using the next-generation matrix. In epidemiology, the next generation matrix is used to derive the Basic Reproduction Number for a compartmental model of the spread of infectious diseases (Roberts \& Heesterbeek, 2013). In population dynamics, it is used to compute the Basic Reproduction Number for a structured population model. It is also used in multi-type branching models for analogous computations.

Let $F(x)$ define as the rate of new infection and $V_{i}(x)$ as the rate of individual displacement, then it defines:

$$
\begin{aligned}
& F=\left[\begin{array}{c}
\frac{\beta I S}{N_{\sigma}} \\
0
\end{array}\right] \\
& V=\left[\begin{array}{c}
(\gamma+\mu) E \\
\left(\theta+\delta_{1}+\mu\right) I-\gamma E
\end{array}\right] \\
& \text { Jacobian } F=\bar{F}=\left[\begin{array}{cc}
0 & \frac{\beta I S}{N_{\sigma}} \\
0 & 0
\end{array}\right] \\
& \text { Jacobian } V=\bar{V}=\left[\begin{array}{cc}
\mu+\gamma & 0 \\
-\gamma & \mu+\delta+\theta
\end{array}\right] \\
& \bar{F} \bar{V}^{-1}=\left[\begin{array}{cc}
1 & 0 \\
\frac{\gamma+\gamma}{(\mu+\gamma)(\mu+\delta+\theta)} & \frac{1}{\mu+\delta+\theta}
\end{array}\right]
\end{aligned}
$$

The eigenvalue of $\bar{F} \bar{V}^{-1}=\frac{\beta S^{0} \gamma}{N_{\sigma}(\mu+\gamma)(\mu+\delta+\theta)}$

with $S^{0}=\frac{r N_{\sigma}}{\mu}$

Then: $R_{0}=\frac{r \beta \gamma}{\mu(\mu+\gamma)(\mu+\delta+\theta)}$

DFE state is defined as the point at which no disease is present in the population. This DFE is the average number of cases of an infectious disease arising by transmission from a single infected individual in a population that has not previously encountered the disease. In other words, it is $R_{0}$. DFE state can be 
achieved if $R_{0}$ is less than one $\left(R_{0}<1\right)$.

Theorem 1. If it is $R_{0}<1$, the disease-free equilibrium point of system (1) is locally asymptotically stable. Meanwhile, if it is $R_{0}>1, L^{0}$ is unstable.

Proof:

Define $L^{0}=\left(S^{0}, E^{0}, I^{0}, R^{0}\right) \quad$ with $S^{0}=\frac{r N_{\sigma}}{\mu}$,

$E^{0}=0, I^{0}=0, R^{0}=0$

Jacobian $(\mathrm{J})$ matrix for $L^{0}=$

$$
\left[\begin{array}{cccc}
-\mu & 0 & -\beta \frac{r}{\mu} & 0 \\
0 & -(\gamma+\mu) & \beta \frac{r}{\mu} & 0 \\
0 & \gamma & -(\delta+\mu+\theta) & 0 \\
0 & 0 & \theta & 0
\end{array}\right]
$$

Then, the characteristic equation derived from Equation (10) is as follows:

$$
(\lambda+\mu)^{2}\left(\lambda^{2}+a_{1} \lambda+a_{2}\right)=0
$$

Where,

$$
\begin{aligned}
& a_{1}=2 \mu+\delta+\theta+\gamma \\
& a_{2}=(\mu+\gamma)(\theta+\mu+\delta)\left(1-R_{0}\right)
\end{aligned}
$$

There are two eigenvalues from Equation (11), which have negative real parts, that is $(\lambda+\mu)^{2}=0$. It results in $\lambda_{1}=\lambda_{1}=-\mu$. So, it needs to consider the other two eigenvalues $\left(\lambda^{2}+\alpha_{1} \lambda+\alpha_{2}\right)=0$.

According to the Routh-Hurwitz criteria (Murray, 2011), the eigenvalues of Equation (11) have negative real parts if and only if it is $\alpha_{1}, \alpha_{2}>0$. Using the equation in $\alpha_{2}$ eigenvalues of Equation (11) will be negative if it is $R_{0}<1$. In control system theory, the Routh-Hurwitz stability criterion is a mathematical test that is a necessary and sufficient condition for the stability of a Linear Time Invariant (LTI) control system. The Routh test is an efficient recursive algorithm that English mathematician Edward John Routh proposed in 1876 to determine whether all the roots of the characteristic polynomial of a linear system had negative real parts. German mathematician, Adolf Hurwitz, independently proposed in 1895 to arrange the polynomial coefficients into a square matrix, called the Hurwitz matrix, and showed that the polynomial was stable if and only if the sequence of determinants of its principal submatrices were all positive. The two procedures are equivalent with the Routh test providing a more efficient way to compute the Hurwitz determinants than computing them directly. A polynomial satisfying the Routh-Hurwitz criterion is called a Hurwitz polynomial (Anagnost \& Desoer, 1989).

EE state is when the disease cannot be totally eradicated but remains in the population. For the disease to persist in the population, the susceptible class, exposed or latent class, infected class, and recovered class must not be zero at equilibrium state. In other words, if $L^{*}=\left(S^{8}, E^{*}, I^{*}, R^{*}\right)$ is the endemic equilibrium state, it is $L^{*}=\left(S^{8}, E^{*}, I^{*}, R^{*}\right) \neq(0,0,0,0)$.

Theorem 2. If it is $R_{0}<1$, the endemic equilibrium point of system in Equation (1) is locally asymptotically stable. Meanwhile, if it is $R_{0}<1, L^{*}$ is unstable.

Proof

Define:

$$
L^{*}=\left(S^{*}, E^{*}, I^{* /}, R^{*}\right)
$$

Where:

$$
\begin{aligned}
& S^{*}=\frac{(\mu+\gamma)(\theta+\mu+\delta) N_{\sigma}}{\beta \gamma} \\
& E^{*}=\frac{\left(R_{0}-1\right)(\theta+\mu+\delta) \mu N_{\sigma}}{\beta \gamma} \\
& I^{*}=\frac{\left(R_{0}-1\right) \mu N_{\sigma}}{\beta} \\
& R^{*}=\frac{\left(R_{0}-1\right) \theta N_{\sigma}}{\beta}
\end{aligned}
$$

Jacobian matrix for $L^{*}=$

$$
\left[\begin{array}{cccc}
-\frac{\beta I^{*}}{N_{\sigma}} & 0 & -\frac{\beta S^{*}}{N_{\sigma}} & 0 \\
\frac{\beta I^{*}}{N_{\sigma}} & -(\gamma+\mu) & \frac{\beta S^{*}}{N_{\sigma}} & 0 \\
0 & \gamma & -(\delta+\mu+\theta) & 0 \\
0 & 0 & \theta & -\mu
\end{array}\right]
$$

Then the characteristic equationderived from Equation (19) is as follows:

$$
(\lambda+\mu)\left(\lambda^{3}+c_{1} \lambda^{2}+c_{2} \lambda+c_{3}\right)=0
$$

Where,

$$
\begin{aligned}
& c_{1}=\beta I^{*}+\delta+\gamma+\theta+3 \mu \\
& c_{2}=3 \mu^{2}+\left(\beta I^{*}+\delta+\gamma+\theta\right) 2 \mu \\
& c_{3}=\left(R_{0}-1\right)(\mu+\gamma)+(\mu+\delta+\theta) \mu
\end{aligned}
$$

There is one of the eigenvalues from Equation (20), which has negative real parts $\lambda_{1}=-\mu$. Then, the research needs to consider the other eigenvalue. The 
eigenvalues in Equation (20) also have negative real parts if and only if it is $c_{1}, c_{2}, c_{3}>0 ; c_{1}, c_{2}>c_{3}$. Using the equation in $c_{3}$ eigenvalues of Equation (5) will be negative if it is $R_{0}>1$.

$$
\left(\lambda^{3}+c_{1} \lambda^{2}+c_{2} \lambda+c_{3}\right)=0
$$

In vector calculus, the Jacobian matrix of a vector-valued function in several variables is the matrix of all its first-order partial derivatives. When this matrix is square, that is, when the function takes the same number of variables as input as the number of vector components of its output, its determinant is referred to as the Jacobian determinant. Both the matrix and the determinant (if it is applicable) are often referred to simply as the Jacobian in literature (Resendis-Antonio, 2013).

Then, optimal control modeling is a common paradigm employed in many fields of science and engineering. Techniques from control theory are used to find the optimal controls that cause the model to behave in a manner that minimizes or maximizes a userdefined performance criterion. To control the outbreak of coronavirus infection, the research applies optimal control method. It aims to minimize susceptible (S) and infected (I) populations, and the value (cost) of vaccination and treatment are represented by $u_{1}$ and $u_{2}$, respectively (Pontryagin, 1962).

Define objective function:

$$
J\left(u_{1}, u_{2}\right)=\min \int_{t_{0}}^{t_{f}} I(t)+\frac{T_{1}}{2} u_{1}^{2}(t)+\frac{T_{1}}{2} u_{2}^{2}(t)
$$

Subject to

$$
\begin{aligned}
& \frac{d S(t)}{d t}=r N_{\sigma}-\frac{\beta I(t) S(t)}{N_{\sigma}}-\mu S(t)-u_{1}(t) S(t) \\
& \frac{d E(t)}{d t}=\frac{\beta I(t) S(t)}{N_{\sigma}}-\gamma E(t)-\mu E(t) \\
& \frac{d I(t)}{d t}=\gamma E(t)-\theta I(t)-\delta I(t)-\mu I(t)-u_{2}(t) I(t) \\
& \frac{d R(t)}{d t}=u_{1}(t) S(t)+u_{2}(t) I(t) \theta I(t)-\mu R(t)
\end{aligned}
$$

with initial condition

$$
S(0)>0, E(0)>0, I(0)>0, R(0)>0
$$

Define:

$$
F\left(I, u_{1}, u_{2}\right)=I(t)+\frac{T_{1}}{2} u_{1}^{2}(t)+\frac{T_{1}}{2} u_{2}^{2}(t)
$$

In here, $T_{1}$ and $T_{2}$ represent the weight constant for control variables of $\mu_{1}$ and $\mu_{2}$, respectively. Then, the terms

$$
\frac{T_{1}}{2} u_{1}^{2}(t) \text { and } \frac{T_{2}}{2} u_{2}^{2}(t)
$$

mean the cost function for vaccine and treatment, respectively. The purpose is to find the control function of $\mu_{1}(t)$ and $\mu_{2}(t)$ with $J\left(u_{l}, u_{2}\right)=\min \left\{\left(u_{l}, u_{2}\right) ; u_{1}, u_{2} \epsilon U\right\}$ subjecting to Equation (6), and the control set is given as follows. That $U=\left\{\left(u_{l}, u_{2}\right) \mid u_{l}(t)\right\}$ is Lebesque measurable on $\left\{[0, T], 0 \leq u_{i}(t)(\leq 1, I=1,2)\right\}$.

For the solution, the optimal control should exist for non-negative initial conditions: positive bounded solutions to the system in Equation (6) and bounded Lebesgue measurable control. There exists an optimal control which minimizes $J\left(u_{1}, u_{2}\right)$ if the following conditions are satisfied. First, $\mathrm{F}$ is a non-empty set, and non-empty set makes sure that there is a solution. Second, the control set, $U$, must be closed and convex.

The right-hand side of the state system is continuous and is bounded above by a linear combination of the control and state. It can be written as a linear function of $U$ with coefficients defined by the time and the state. The integrand of the objective function is convex on $U$.

A controllability matrix of the system is needed to stabilize the system. In addition, solutions to an optimal control problem may not be obtained if the system concerned is not able to be controlled. Thus, it needs to analyze the control of the system. Controllability can be analyzed by forming a control matrix and determining the number of ranks of the matrix. Then, it define the dynamical model of the system as follows.

$$
\dot{x}(t)=A x(t)+B u(t)
$$

The nonlinear controllability matrix is developed from intuitive control problem examples (Whalen, Brennan, Sauer, \& Schiff, 2015). The controllability matrix is a mapping constructed from an input function and its higher-order Lie brackets. The Lie bracket is an algebraic operation on two vector fields $f(x), g(x) \epsilon$ $\mathfrak{R}^{m}$ that creates a third vector field $\mathfrak{I}(x)$. With $g$ as the input control vector $\mathrm{u} \in \mathfrak{R}^{m}$ it defines an embedding in $\mathfrak{R}^{m}$ that maps the input to states. For a nonlinear system, the research replaces $A x(t)$ in Equation (28) by a nonlinear vector field $A_{N Z}(x(t))$, take the input function as $g=B u(t)$ in Equation (28), and create Lie bracket with respect to the nonlinear vector field $f(x$ $(t))=A_{N Z}(x(t))$. The Lie bracket is defined as follows.

$$
\begin{aligned}
& \left(a d_{f}^{1}, g\right)=[f, g]=\frac{\partial g}{\partial x} f-\frac{\partial f}{\partial x} g \\
& \left(a d_{f}^{2}, g\right)=[f,[f, g]]=\frac{\partial\left(a d d_{j} g\right)}{\partial x} f-\frac{\partial f}{\partial x}\left(a d_{f}^{1}, g\right) \\
& \vdots \\
& \left(a d_{f}^{k}, g\right)=\left[f,\left(a d_{f}^{k-1}, g\right)\right]
\end{aligned}
$$

That $\left(a d_{f}^{k}, g\right)$ is the adjoint operator, and the superscripts represent the order of the Lie bracket. With formal definitions of the input function in Equation (27) and its higher Lie brackets in Equation (29) from 1 to $n$, where $n$ is the order of the system matrix $A_{N Z}(x(t))$, the nonlinear controllability matrix is defined as follows. 


$$
\begin{aligned}
& Q \equiv\left[g,\left(d_{f}^{1}, g\right), \ldots,(d \underset{f}{m}, g)\right] \\
& =\left[g,[f, g],[f[f, g]], \ldots,\left[f,\left(d_{f}^{n-1}, g\right)\right]\right.
\end{aligned}
$$

After calculation using Maple software programming, it is known that the rank of controllability matrix equals to four. It means that the dynamical system of the model is controllable. Then, the formulation of optimal control is applied to find the optimal solution. After that, the research defines the Hamiltonian function of the control as follows.

$$
H=I(t)+\frac{T_{1}}{2} u_{1}^{2}+\frac{T_{1}}{2} u_{1}^{2}+\lambda_{1} \dot{S}+\lambda_{2} \dot{E}+\lambda_{3} \dot{I}+\lambda_{4} \dot{R}
$$

Here, $\lambda_{1}, \lambda_{3}, \lambda_{3}, \lambda_{4}$ are adjoint variables that satisfy:

$$
\begin{aligned}
& \frac{d \lambda_{1}}{d t}=-\frac{d H}{d S}=-1+\lambda_{1}\left(\mu+u_{1}\right)-\lambda_{1} u_{1}-\frac{\beta I\left(\lambda_{1}-\lambda_{2}\right)}{N_{\sigma}} \\
& \frac{d \lambda_{2}}{d t}=-\frac{d H}{d E}=\lambda_{2}(\mu+\gamma)-\lambda_{3} \gamma \\
& \frac{d \lambda_{3}}{d t}=-\frac{d H}{d I}=-1+\lambda_{3}\left(\mu+\delta+\theta+u_{2}\right)-\lambda_{1}\left(u_{2}+\theta\right)-\frac{\beta S\left(\lambda_{2}-\lambda_{1}\right)}{N_{\sigma}} \\
& \frac{d \lambda_{4}}{d t}=\frac{d H}{d R}=\lambda_{4} \mu
\end{aligned}
$$

With terminal condition:

$$
\lambda_{1}\left(t_{f}\right)=\lambda_{2}\left(t_{f}\right)=\lambda_{3}\left(t_{f}\right)=\lambda_{4}\left(t_{f}\right)=0
$$

These adjoint variables will maximize or minimize the state variable with respect to the state function. Then the optimal variables of control are as follows.

$$
\frac{d H}{d u_{1}}=S\left(\lambda_{4}-\lambda_{1}\right)+T_{1} u_{1}=0 \Rightarrow u_{1}^{*}=\frac{S\left(\lambda_{1}-\lambda_{4}\right)}{T_{1}}
$$

with $u_{1}=u_{1}^{*}$

$$
\frac{d H}{d u_{2}}=I\left(\lambda_{4}-\lambda_{3}\right)+T_{2} u_{2}=0 \Rightarrow u_{2}^{*}=\frac{I\left(\lambda_{3}-\lambda_{4}\right)}{T_{2}}
$$

with $u_{2}=u_{2}^{*}$

Then, $u_{1}{ }^{*}$ can be rewritten as:

$$
u_{1}^{*}=\max \left\{0, \min \left(\frac{S\left(\lambda_{1}-\lambda_{4}\right)}{T_{1}}, 1\right)\right\}
$$

and $u_{2}^{*}$

$$
u_{2}^{*}=\max \left\{0, \min \left(\frac{I\left(\lambda_{3}-\lambda_{4}\right)}{T_{2}}, 1\right)\right\}
$$

Numerical results and analysis of the optimal control are performed with GNU Octave software version 5.2.0 (Eaton et al., 2021). It uses a forwardbackward sweep of the fourth-order Runge Kutta method. The forward sweep method is for solving the state in Equation (6), and backward sweep method solves adjoint variable in Equation (9) (Anita et al., 2011).

GNU Octave is a high-level language primarily intended for numerical computations. It is typically used for solving linear and nonlinear equations, numerical linear algebra, and statistical analysis and performing other numerical experiments. It may also be used as a batch-oriented language for automated data processing (Eaton et al., 2021).

Octave is free software. It means that everyone is free to use and redistribute it on certain conditions. Octave is not, however, in the public domain. It

Table 1 Parameter Data of Equations (1) and (6).

\begin{tabular}{cccc}
\hline Parameter & Description & Values & Reference \\
\hline$\beta$ & Transmission coefficient & 0,7873 & Kan et al. (2005) \\
$N_{\sigma}$ & Population equal with $S(0)$ & 258.639 & Al Farizi and Harmawan (2020) \\
$\gamma$ & Rate from latent to infected & 3 to 7 & Kan et al. (2005) \\
$\delta$ & Mortality rate due to infection & 0,154 & Kan et al. (2005) \\
$\theta$ & Rate from infected to recovery & 0,84 & Kan et al. (2005) \\
$r$ & Source of susceptible rate & 0,0452 & Fitted \\
$\mu$ & Natural death rate & 0,0016 & Fitted \\
$S(0)$ & Initial value of $S(t)$ & 258.639 & Al Farizi and Harmawan (2020) \\
$E(0)$ & Initial value of $E(t)$ & 33.672 & Al Farizi and Harmawan (2020) \\
$I(0)$ & Initial value of $I(t)$ & 16.006 & Al Farizi and Harmawan (2020) \\
$R(0)$ & Initial value of $R(t)$ & 3.518 & Al Farizi and Harmawan (2020) \\
$T_{1}$ & Weight constant of $U_{1}$ & 1 & Fitted \\
$T_{2}$ & Weight constant of $U_{2}$ & 1 & Fitted \\
\hline
\end{tabular}


is copyrighted, and there are restrictions on its distribution, but the restrictions are designed to ensure that others will have the same freedom to use and redistribute Octave. The precise conditions can be found in the GNU General Public License that comes with Octave (Eaton et al., 2021).

The parameter data of the system in Equations (1) and (6) are presented in Table 1. Then, the terminal condition is $\lambda_{i}\left(t_{f}\right)=0, i=1,2,3,4$. The research puts 200 days $\left(t_{f}=200\right)$ as the timeline for the dynamical system.

Based on Table 1, $R_{0}$ which is simulated in the dynamical system of Equation (1) is 22.339. It means that through the calculation, the value of $R_{0}$ equals 22.339. Then, the value of the parameter data of susceptible rate $(r)$ and parameter data of natural death rate $(\mu)$ are fitted or adjusted. After the data of $r$ and $\mu$ are fitted and adjusted, the simulation model can be run using GNU Octave software programming.

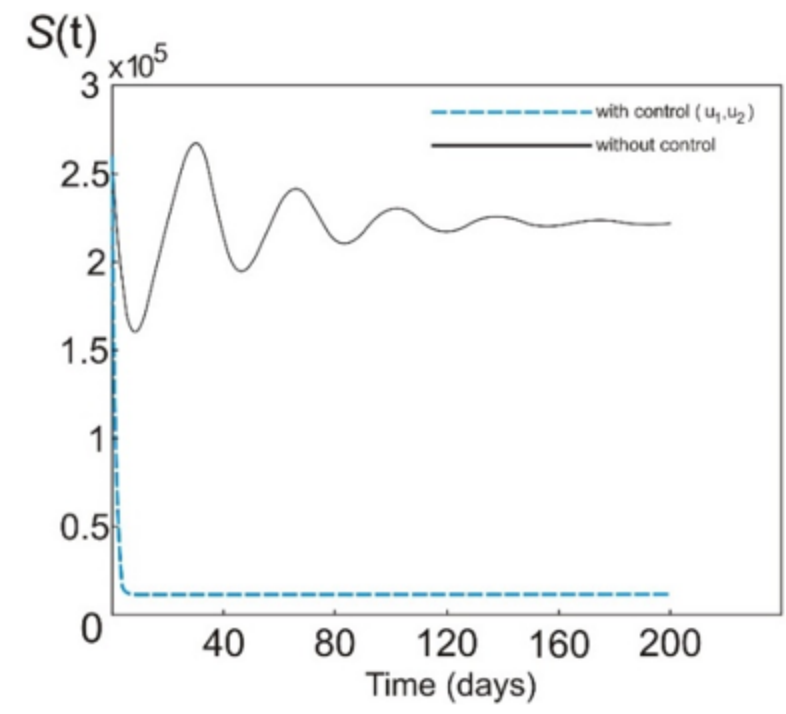

Figure 2 Dynamic of Susceptible Population

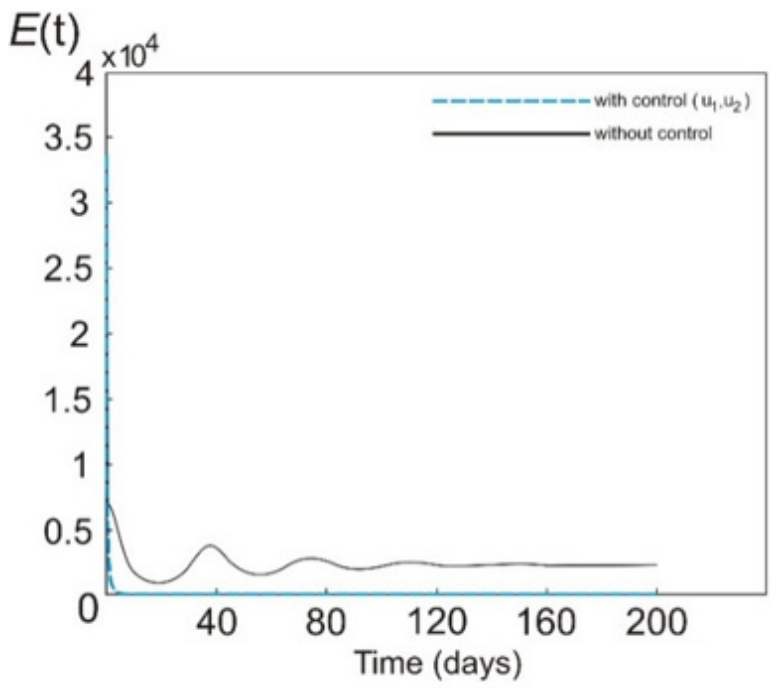

Figure 3 Dynamic of Exposed Population

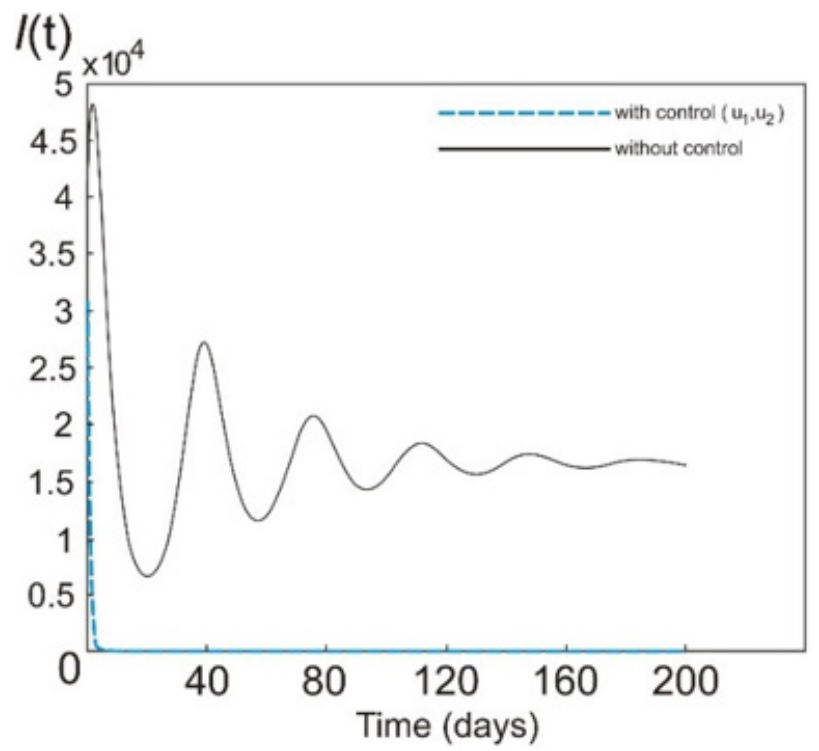

Figure 4 Dynamic of Infected Population

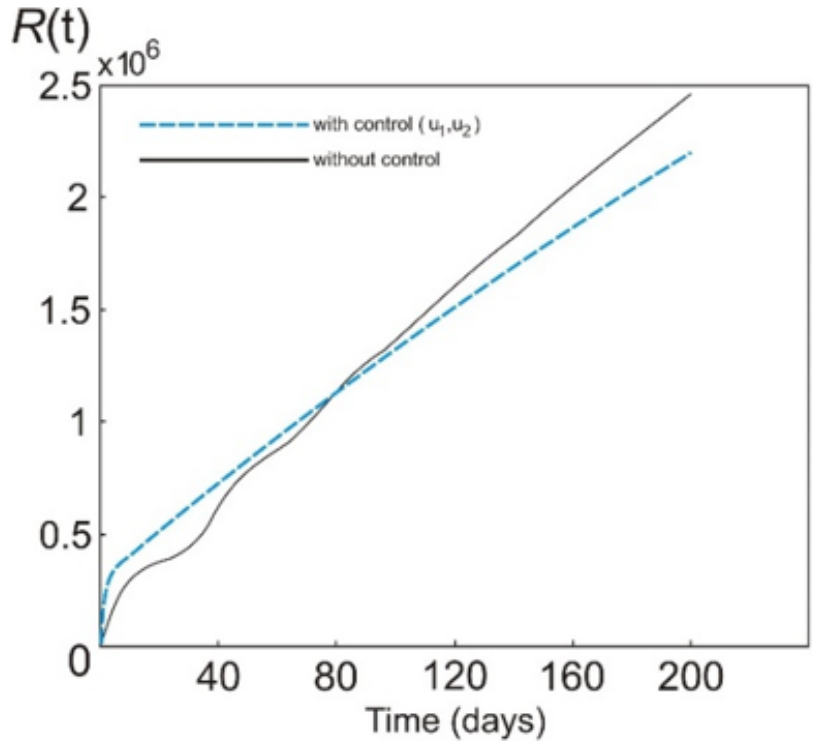

Figure 5 Dynamic of Recovered Population

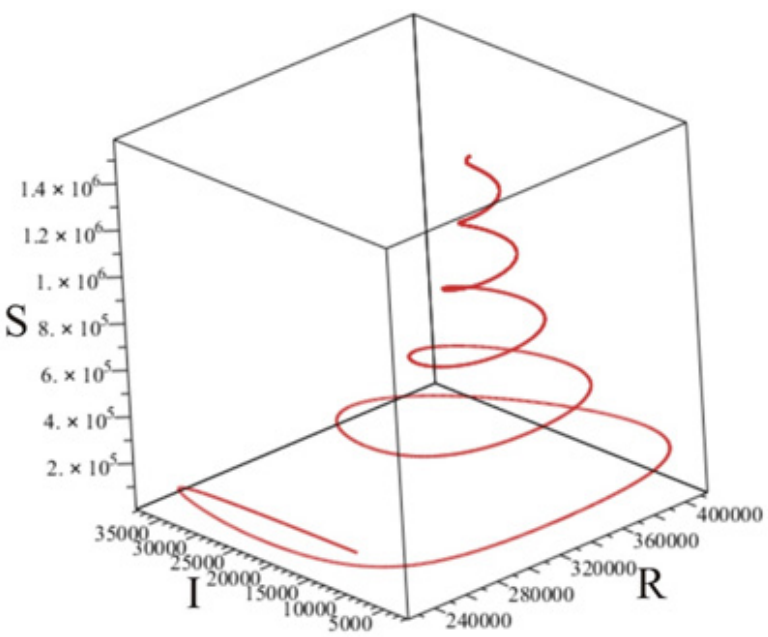

Figure 6 Three-Dimensional Graph of Dynamic Population of $\mathrm{S}, \mathrm{I}, \mathrm{R}$ without Control 
After numerical calculations, the research can see be in the simulation graph in Figures 2 to 5 that the control function of $u_{1}$ and $u_{2}$ (vaccination and treatment) are applied to the system. Then, the control function can significantly decrease the population of susceptible (see Figure 2). It also minimizes the population of infected (see Figure 4).

Figure 6 describes the three-dimensional (3D) phase between the number of susceptible, infectious, and recovered people as the variable. This 3D phase can also be described as a graph of parametric equation between susceptible, infectious, and recovered.

The terms of $-u_{1}(t) S(t)$ and $u_{1}(t) S(t)$ in the system in Equation (6) means that there is a reduction in the rate of change of the susceptible population by $u_{1}(t) S(t)$ and the infected population as much as $u_{2}(t)$ $I(t)$. Using GNU Octave software programming, it is known that $S(3)=170.100, u_{1}(3)=0,9999 ; I(3)=28.820$; $u_{2}(3)=0,9998$ with $t=3$ months so that $S(3) \times u_{1}(3)$ $=170.082,99$ and $I(3) \times u_{2}(3)=28.814,236$. It means when the rate of the susceptible population at $t=3$ months $(S(3))$ must be reduced by 170.083 individuals (rounding up from 170.082,99). Then, when the rate of the infected population at $t=3$ months, $(I(3))$ must be reduced by 28.815 individuals (rounding up from $28.814,236)$ simultaneously. For a further description, in the third month, it shows the target to vaccinate 170.083 susceptible individuals and treat 28.815 infected individuals simultaneously. The susceptible, exposed, and infected population will decrease rather than not being vaccinated and treated when it is done. These vaccination and treatments should continue for 200 days with notes that the amount of $u_{1}(t) S(t)$ and $u_{2}(t) I(t)$ will vary depending on time $(t)$. It depends on numerical calculations of the dynamical system in Equations (6) and (9). It also notes that the value of $u_{1}$ and $u_{2}$ is constrained to $0<u_{1}<1,0<u_{2}<1$.

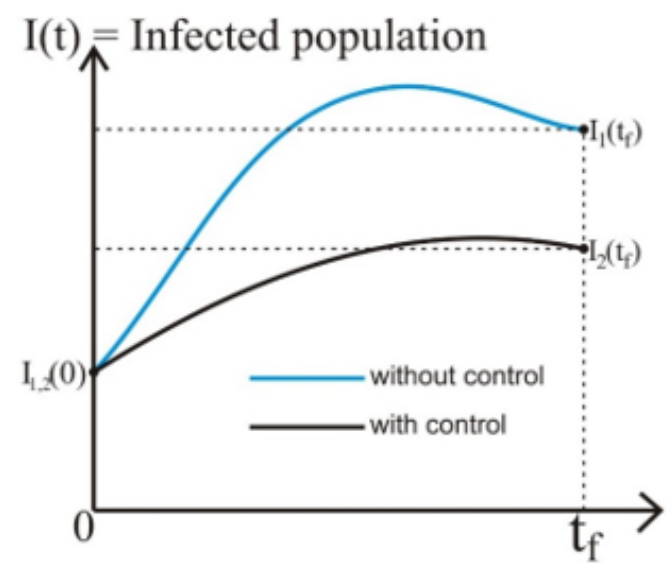

Figure 7 Dynamics of Infected Population with and without Controls

The depiction of the use of the control in the research can be seen in Figure 7. The use of this control is expected to reduce the infected population at the specified time, $t_{f}$. As seen in Figure 7, the blue line is the equation of infected individual versus time without control and reaches final state at $I_{1}\left(\mathrm{t}_{\mathrm{f}}\right)$. Then, the black line is the equation of infected individual versus time with control and reaches final state at $I_{2}\left(\mathrm{t}_{\mathrm{f}}\right)$. It also shows the value of the final state $I_{2}\left(t_{f}\right)<I_{1}^{2}\left(t_{f}\right)$ . It means that the control can decrease the final state. The type of this optimal control problem is fixed time with free endpoint and state and control constraint.

\section{CONCLUSIONS}

The use of $u_{1}$ and $u_{2}$ as controls applied to the dynamical modeling of coronavirus spread can be seen in the numerical simulation in Figures 2, 3, 4, and 5. There is a reduction in the number of susceptible and infected populations. It means that giving vaccines to susceptible populations and treating infected populations have an impact or effect on reducing the number of the susceptible population and infected population. It also implies that this control can reduce the spread of the virus. In the end, the use of control measures is expected to advise the public health authorities or government to optimize the cost or value of vaccines and treatments to handle cases of COVID-19 outbreak.

The research is limited to four population systems (susceptible, exposed, infectious, and recovered) and uses only the population in Indonesia. For further research, it can add more population systems, such as quarantined populations. Future research can also divide the infectious population into two classes, like the early-stage infection and chronic infection population, to describe more detail about the COVID-19 outbreak.

\section{REFERENCES}

Anagnost, J. J., \& Desoer, C. A. (1989). An elementary proof of the Routh-Hurwitz stability criterion (Technical report). University of California.

Annas, S., Pratama, M. I., Rifandi, M., Sanusi, W., \& Side, S. (2020). Stability analysis and numerical simulation of SEIR model for pandemic COVID-19 spread in Indonesia. Chaos, Solitons \& Fractals, 139(October), 1-19. https://doi.org/10.1016/j. chaos.2020.110072

Anita, S., Arnăutu, V., \& Capasso, V. (2011). An introduction to optimal control problems in life sciences and economics: From mathematical models to numerical simulation with MATLAB ${ }^{\circledR}$. Springer Science+Business Media.

Brauer, F., \& Castillo-Chavez, C. (2013). Mathematical models in population biology and epidemiology. Springer Science+Business Media.

Al Farizi, S., \& Harmawan, B. N. (2020). Data transparency and information sharing: Coronavirus prevention problems in Indonesia. Jurnal Administrasi Kesehatan Indonesia, 8, 35-50. https://doi. org/10.20473/jaki.v8i2.2020.35-50 
Delamater, P. L., Street, E. J., Leslie, T. F., Yang, Y. T., \& Jacobsen, K. H. (2019). Complexity of the basic reproduction number (R0). Emerging Infectious Diseases, 25(1), 1-4. https://doi.org/10.3201/ eid2501.171901

Eaton, J. W., Bateman, D., Hauberg, S., \& Wehbring, R. (2021). GNU Octave: A high-level interactive language for numerical computations. Retrieved from https://enacit.epfl.ch/cours/matlab-octave/ octave-documentation/octave/octave.pdf

Hattaf, K., \& Dutta, H. (Eds.) (2020). Mathematical modelling and analysis of infectious diseases. Springer.

Kan, B., Wang, M., Jing, H., Xu, H., Jiang, X., Yan, M., ... \& Xu, J. (2005). Molecular evolution analysis and geographic investigation of severe acute respiratory syndrome coronavirus-like virus in palm civets at an animal market and on farms. Journal of Virology, 79(18), 11892-11900. https://doi.org/10.1128/ jvi.79.18.11892-11900.2005

Krishna, M. V., \& Prakash, J. (2020). Mathematical modelling on phase based transmissibility of coronavirus. Infectious Disease Modelling, 5, 375385. https://doi.org/10.1016/j.idm.2020.06.005

Kumar, V., \& Kumar, D. (2018). Analysis of epidemic model using basic reproduction number. In Proceedings of $3^{\text {rd }}$ International Conference on Internet of Things and Connected Technologies (ICIoTCT) (pp. 26-27). https://doi.org/10.2139/ssrn.3166500

Li, H., Chen, X., \& Huang, H. (2020). The novel coronavirus outbreak: What can be learned from China in public reporting? Global Health Research and Policy, 5, 1-3. https://doi.org/10.1186/s41256-020-00140-9

Murray, J. D. (2011). Mathematical biology: I. An introduction. Springer.

Pontryagin, L. S. (1962). The mathematical theory of optimal processes. Interscience Publishers.

Resendis-Antonio, O. (2013). Jacobian matrix. In W. Dubitzky, O. Wolkenhauer, K. H. Cho, \& H. Yokota (Eds.), Encyclopedia of systems biology. Springer. https://doi.org/10.1007/978-1-4419-9863-7_1367
Roberts, M. G., \& Heesterbeek, J. A. P. (2013). Characterizing the next-generation matrix and basic reproduction number in ecological epidemiology. Journal of Mathematical Biology, 66, 1045-1064. https://doi.org/10.1007/s00285-012-0602-1

Shereen, M. A., Khan, S., Kazmi, A., Bashir, N., \& Siddique, R. (2020). COVID-19 infection: Emergence, transmission, and characteristics of human coronaviruses. Journal of Advanced Research, 24(July), 91-98. https://doi.org/10.1016/j. jare.2020.03.005

Van den Driessche, P. (2017). Reproduction numbers of infectious disease models. Infectious Disease Modelling, 2(3), 288-303. https://doi.org/10.1016/j. idm.2017.06.002

Wang, C., Horby, P. W., Hayden, F. G., \& Gao, G. F. (2020). A novel coronavirus outbreak of global health concern. The Lancet, 395(10223), 470-473. https:// doi.org/10.1016/s0140-6736(20)30185-9

Wang, M., \& Hu, Z. (2013). Bats as animal reservoirs for the SARS coronavirus: Hypothesis proved after 10 years of virus hunting. Virologica Sinica, 28, 315317. https://doi.org/10.1007/s12250-013-3402-x

Whalen, A. J., Brennan, S. N., Sauer, T. D., \& Schiff, S. J. (2015). Observability and controllability of nonlinear networks: The role of symmetry. Physical Review $X, 5(1)$, 1-18. https://doi.org/10.1103/ physrevx.5.011005

Yang, Z., Zeng, Z., Wang, K., Wong, S. S., Liang, W., Zanin, M., ... \& He, J. (2020). Modified SEIR and AI prediction of the epidemics trend of COVID-19 in China under public health interventions. Journal of thoracic disease, 12(3), 165-174. https://doi. org/10.21037/jtd.2020.02.64

Zheng, B. J., Guan, Y., Wong, K. H., Zhou, J., Wong, K. L., Young, B. W. Y., ... \& Lee, S. S. (2004). SARSrelated virus predating SARS outbreak, Hong Kong. Emerging Infectious Diseases, 10(2), 176-178. https://doi.org/10.3201/eid1002.030533 